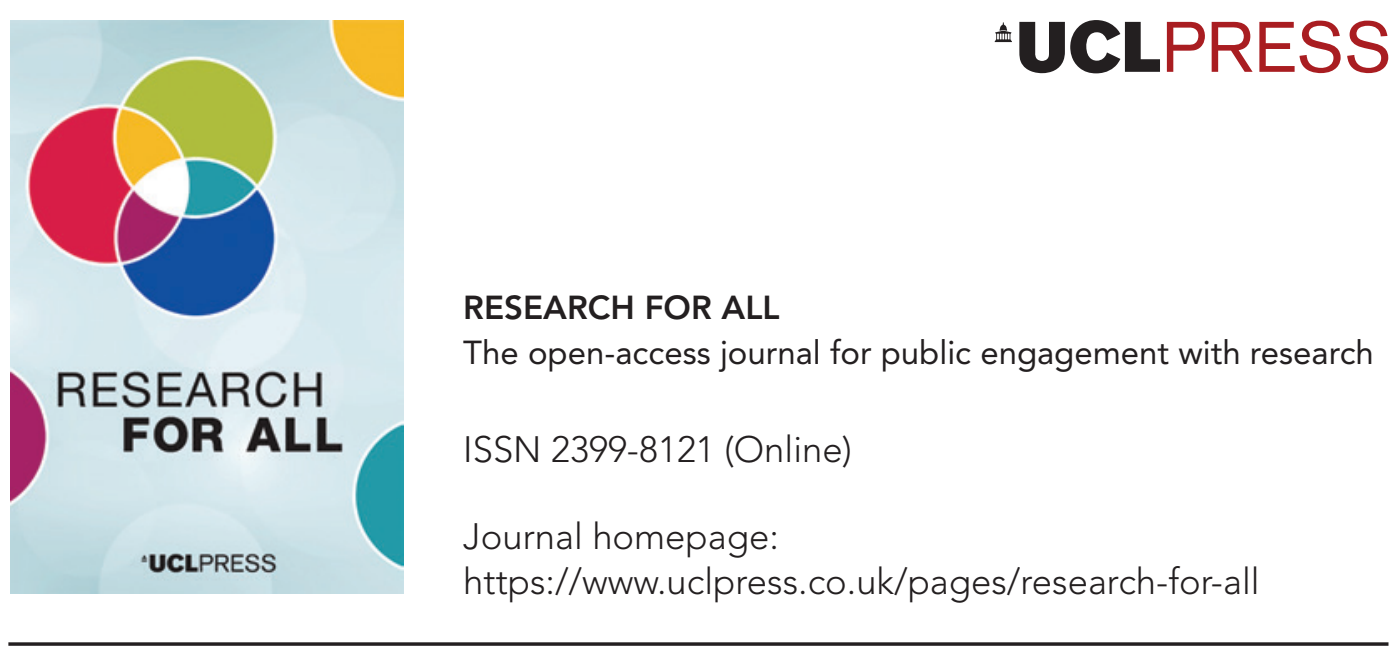

\title{
Developing arts-based methods for exploring virtual reality technologies: A university-industry case study
}

Stuart Gray, Chris Bevan, Kirsten Cater, Jo Gildersleve, Caroline Garland and Oliver Langdon

\section{How to cite this article}

Gray, S., Bevan, C., Cater, K., Gildersleve, J., Garland, C. and Langdon, O. (2021) 'Developing arts-based methods for exploring virtual reality technologies: A universityindustry case study'. Research for All, 5 (2), 246-70. https://doi.org/10.14324/RFA.05.2.05

Submission date: 14 September 2020

Acceptance date: 4 May 2021

Publication date: 21 September 2021

\section{Peer review}

This article has been peer-reviewed through the journal's standard double-blind peer review, where both the reviewers and authors are anonymized during review.

\section{Copyright}

(C) 2021 Gray, Bevan, Cater, Gildersleve, Garland and Langdon. This is an open-access article distributed under the terms of the Creative Commons Attribution Licence (CC BY) 4.0 https:// creativecommons.org/licenses/by/4.0/, which permits unrestricted use, distribution and reproduction in any medium, provided the original authors and source are credited.

\section{Open access}

Research for All is a peer-reviewed open-access journal. 


\title{
Developing arts-based methods for exploring virtual reality technologies: A university-industry case study
}

\author{
Stuart Gray*, Chris Bevan, Kirsten Cater, Jo Gildersleve - University of \\ Bristol, UK \\ Caroline Garland, Oliver Langdon - Kilter Theatre, Bath, UK
}

\begin{abstract}
Collaborations between human-computer interaction $(\mathrm{HCl})$ researchers and arts practitioners frequently centre on the development of creative content using novel - often emergent - technologies. Concurrently, many of the techniques that $\mathrm{HCl}$ researchers use in evaluative participant-based research have their roots in the arts - such as sketching, writing, artefact prototyping and role play. In this reflective paper, we describe a recent collaboration between a group of $\mathrm{HCl}$ researchers and dramatists from the immersive theatre organization Kilter, who worked together to design a series of audience-based interventions to explore the ethics of virtual reality (VR) technology. Through a process of knowledge exchange, the collaboration provided the researchers with new techniques to explore, ideate and communicate their work, and provided the dramatists with a solid academic grounding in order to produce an accurate yet provocative piece of theatrically based design fiction. We describe the formation of this partnership between academia and creative industry, document our journey together, and share the lasting impact it has had upon both parties.
\end{abstract}

Keywords: university-industry collaboration, human-computer interaction, participatory methods, immersive theatre, design fiction, virtual reality

\section{Key messages}

- The dramatic arts offer technology researchers a range of novel techniques for facilitating ideation and open discussion around complex future/emergent technologies with young adults.

- Despite overlap in methods and terminology between academic and creative industry partners, organizational differences in culture, procedure and structure are a leading cause of friction, and can consume a great deal of time within a knowledge exchange collaboration if they are not identified and mitigated early.

- While the arts heavily influence many participatory methods in the field of human-computer interaction, differences may exist with regard to the responsibilities of the actors involved, as well as what constitutes impactful output. 


\section{Introduction}

Since the early 1960s, human-computer interaction $(\mathrm{HCl})$ has established itself as a people-centred research discipline at the intersection of computer science, design, cognitive science and human factors (for example, Fitzpatrick, 2018; Myers, 1998). From its inception, $\mathrm{HCl}$ has actively sought and embraced interdisciplinarity (for example, Blackwell, 2015; Rogers, 2012), and researchers in the field continue to collaborate with an ever-increasing range of academic, industrial and creative industry partners to pioneer new technologies (Chilana et al., 2015), propagate technological theory (Rogers, 2012) and establish innovative research methods (Shneiderman, 2011).

The use of artistic methods to support research interventions - especially with emergent technologies and their associated social impacts - has resulted in a long-standing symbiotic relationship between $\mathrm{HCl}$ researchers and arts creatives (for example, Laurel, 2013; Jeon et al., 2019). Artistic methods such as drawing, the creation of physical objects and role play have been borrowed from the arts and been used by academic researchers to convey complex technical concepts to other academics and the general public alike. Artistic methods can also help facilitate research participants to better express their views and requirements, and serve as artefacts which can explain and inform technology design (Duarte and Baranauskas, 2018; Edmonds, 2017). Despite this, however, few $\mathrm{HCl}$ researchers have formal training in artistic methods, with the majority having come to the field from science, technology, engineering and mathematics (STEM) or psychology backgrounds.

Compounding this, many of the traditional avenues used to exhibit $\mathrm{HCl}$ research outputs - journal papers, articles and conference presentations - prioritize the scientific merits of the work, and these can sometimes overlook the impact of artistic endeavours (Sengers and Csíkszentmihályi, 2003). While niche venues for unconventional artistic methods and viewpoints do exist in $\mathrm{HCl}$ (for example, alt.chi (Ames et al., 2015)), any discourse they may provoke is often limited to $\mathrm{HCl}$ academics and industry professionals.

One alternative, and increasingly common, means of supporting knowledge and skill acquisition, and generating research impact for $\mathrm{HCl}$ academics, is through university-industry knowledge exchange collaboration. Such collaborations offer each partner access to new audiences and the opportunity to disseminate their work through new channels.

In their 'triple helix' model of university-industry-government relations, Etzkowitz and Leydesdorff (2000) position knowledge exchange alongside teaching and research as a core mission of the university as an institution. Johnson (2020) provides a recent overview of the UK's view on the importance of knowledge exchange, including the Knowledge Exchange Framework (KEF) through which it measures the effectiveness of its investment in this area (Research England, 2021). Drawing mainly from case studies, a number of recent works have begun to formulate best practices for successful university-industry knowledge exchange partnerships. The UK Economic and Social Research Council, for example, provides a number of practical tips for conducting and evaluating knowledge exchange (ESRC, 2021), and Crawley et al. (2020) offer a systematic approach to knowledge exchange that identifies three key actions that are important to success:

1) Careful identification of relevant partners, followed by a bidirectional discussion of the needs of society, the partners and the university

2) Sensitivity and responsiveness to these needs in the conduct of education, research and catalysing innovation 
3) A proactive process that exchanges the talented graduates, discoveries and creation outcomes of the university, and assists in their uptake by the partners.

However, no model of successful knowledge exchange collaboration is 'one size fits all'. Of particular relevance to the present case study, partnerships between universities and the creative sector, for example, have their own idiosyncratic characteristics (for example, Sedgman, 2019). One such characteristic, as Williams et al. (2017: 6) highlight, is that these collaborations are more often than not bidirectional: 'They exhibit mutuality and are primarily conducted through shared investigation rather than transfer from an "expert" institution to the industry context.' There are limited frameworks available to guide $\mathrm{HCl}$ academics and creative sector collaborators towards successful partnership outcomes. Moreover, many of the publications produced as a result of such collaborations focus almost exclusively upon the design processes and outcomes of the novel innovations produced, rather than representing and reflecting upon the wider journey of the partnership.

In this paper, we present a case study of a collaborative exploration of virtual reality $(\mathrm{VR})$ technologies between a group of $\mathrm{HCl}$ researchers based at the University of Bristol and dramatists from the immersive theatre company Kilter. The collaboration considered how immersive theatre techniques might be used to better elicit audience participation in the ideation of the possible ethical concerns raised by VR, building upon potential issues raised in recent literature (for example, Madary and Metzinger, 2016; Spiegel, 2018). Further, we pay particular attention to the involvement of young people - the designers and users of the future, who may be left to deal with any consequences of the decisions taken about the design of VR today.

First, we contribute an overview of the use of arts-based methods within $\mathrm{HCl}$, and some known factors in successful university-industry partnerships, before presenting our case study collaboration. This begins with a description of the early relationship building between the $\mathrm{HCl}$ and Kilter teams, before describing the core activities undertaken within the collaboration: (1) three ideation workshops with 12 young people from an English secondary school, which aimed to better understand the ethical concerns of future VR technologies; and (2) VR100, a bespoke immersive theatre production, which was inspired by the views of the young people and developed as an output of the collaboration to connect our VR research with public audiences. Finally, we reflect upon the impact that the collaboration has had for the partnering organizations, before sharing and discussing five valuable insights applicable to future collaborations between $\mathrm{HCl}$ academics and industry.

\section{Background: The use of arts-based methods within participatory $\mathrm{HCl}$ research}

In $\mathrm{HCl}$ research, a range of arts-based methods are used to support participatory approaches to the design and evaluation of new and emerging technologies. During the design process, a constructivist approach is often followed, which challenges participants to learn about technologies through the making of physical artefacts, before using these creatively to elaborate upon primitive designs. Building artefacts that represent and characterize technologies forms a common language for expressing ideas; they allow participants to physically demonstrate to researchers what - they feel a technology is, and how it might be used (Ehn, 1988). This process is often iterative, with different techniques implemented to increase the sophistication of designs over time. For instance, techniques such as paper-based prototyping (for example, Sefelin et al., 2003) and wireframing (for example, Marquardt, 2017) are often used for early 
low-fidelity prototypes, before evolving into constructions with greater material and technical complexity. Similarly, design sketching (for example, Sturdee and Lindley, 2019) can be used to elicit and explore participants' thought processes to portray visuospatial ideas about the aesthetic of a technology and a user's potential experience of it (Sefelin et al., 2003; Tversky, 2002).

Techniques rooted in the performance arts have also been used to inform the design of $\mathrm{HCl}$ research interventions. Role play, for example, grants participants an alternative and often highly engaging route by which ideas can be represented and understood (Svanaes and Seland, 2004; Sato and Salvador, 1999), and this can be undertaken with varying degrees of dramatism (Seland, 2009, 2010; Newell et al., 2006). Within $\mathrm{HCl}$, role play has been used to simulate technology-mediated scenarios, allowing researchers to observe participants as they use an existing technology or prototype. It can also be used to improvise functionality that is beyond the current capabilities of an artefact (Schepers et al., 2018). Frequently, however, role play is facilitated by researchers who do not themselves have any formal dramatic training.

Finally, design fiction, a relatively recent addition to $\mathrm{HCl}$ research methods, involves supporting participants during a design or ideation process to visualize a fictional future world in which a technology has brought about palpable change (Bleecker, 2009; Levine, 2017). In design fiction 'diegetic prototypes [are used] to suspend disbelief about change' (Sterling, 2013: n.p.). While traditional technology prototyping posits a model or representation of a possible future technology for today's world, a diegetic prototype portrays it as a functional technology within a fictional world. In other words, carefully designed artefacts (for example, physical objects or other media such as advertisements or news reports) are used by researchers to create a cohesive narrative of a fictional world within which participants can be immersed (Bleecker, 2009).

In many respects, design fiction is a theatrical operationalization of science fiction for the purposes of research. Through immersing participants within a fictional world, the technique allows researchers to understand the role a technology plays within it - not just its affordances, but also its implications for users and wider society. Questions can therefore be elicited and explored across a wide gamut, from functional intrigue, such as how a technology works and what the user experience may entail, to more abstract concerns such as ethics (Bleecker, 2009).

Design fiction invites multiple forms of artistry in the modelling of its artefacts, and the technique is most effective when these artefacts portray a rich and compelling creative narrative, involving 'dialogue, plot rationalizations, character interactions and narrative structure' (Kirby, 2010: 41; Bleecker, 2009). Thus, the affordances, issues and impact of a technology are unveiled as it mediates fictional character interactions with others and their world. To truly immerse participants, considerable artistic skill is required. Design fictions may be presented using a range of media - including tangible prototypes (for example, Sturdee et al., 2017); technical documentation (Lindley and Coulton, 2016); paper-based media (for example, comics, newspapers and magazines) (Coulton et al., 2017); digital imagery and videos (Lindley and Potts, 2014); and virtual environments (Green et al., 2019).

\section{Understanding the drivers of success within academic-industry partnerships}

Given its long-established interdisciplinarity and propensity to explore novel innovation, academics in $\mathrm{HCl}$ frequently work closely with industry for research, development and knowledge exchange. Despite this, little collaboration modelling has been undertaken 
with $\mathrm{HCl}$ academics and their industry partners in order to understand the successful ingredients of such partnerships. Furthermore, while heuristics for successful collaboration have been produced by academics from other fields, it is unclear how well they may generalize to $\mathrm{HCl}$. However, from their recent systematic review of what makes industry-university collaborations succeed more generally, Rybnicek and Königsgruber (2019) outline a model for success that is based on three general factors: flexibility, honesty and clarity.

The first factor, flexibility, considers the expectations and availability of resources that each partner can offer the other - including time (Goduscheit and Knudsen, 2015), infrastructure (Arvanitis et al., 2008; Boardman and Bozeman, 2015) and expertise (Myoken, 2013). This factor also considers acting to ease the challenges of different institutional structures and cultures (Boardman and Bozeman, 2015; Schofield, 2013) by each partner educating the other about their institutional environment (Barnes et al., 2002), and developing shared conflict avoidance protocols (Muscio and Vallanti, 2014). The model advocates for both partners exhibiting a willingness to compromise and change where possible (Muscio and Vallanti, 2014), but without sacrificing core values (Starbuck, 2001).

The second factor, honesty, is underpinned by cultivating varied and reciprocal channels of communication (Bstieler et al., 2017) to create shared understanding and objectives (Hong et al., 2010; Lee, 2011). This factor identifies the risks caused by diverging goals (Henderson et al., 2006), and instead argues for clearly stated shared and individual organizational goals and interests which both teams commit to understand and support (Attia, 2015; Canhoto et al., 2016) - thus creating a winwin situation (Lai and Lu, 2016). This can only be done, however, through interorganizational transparency at the beginning of the partnership to create a foundation of trust and openness to speak openly about inter-organizational challenges (Schein, 2010). Moreover, this factor proposes the significance of knowledge and technology transfer in fostering innovation (Guan et al., 2005; MingJi and Ping, 2014).

Finally, clarity - having an awareness of the framework encapsulating the collaboration - is essential. Being aware of current economic, legal, political or social developments can deeply impact partnership success by bringing external pressure upon each organization (Berbegal-Mirabent et al., 2015; Hadjimanolis, 2006). But this also concerns the internal framework and working environment of the collaboration with issues including the geographical distance between collaborators (Indarti and Wahid, 2013), the frequency of co-working, leadership responsibilities and contractual obligations (Rybnicek and Königsgruber, 2019).

Rybnicek and Königsgruber's (2019) systematic review also highlights that previous work often neglects to consider the contextual specificities of their findings. Thus, they identify four possible modulators of these three factors that remain unexplained in the literature:

1) Scale. Organizations of different scales (for example, small- and medium-sized enterprises, and multinationals) have different requirements, flexibilities and viewpoints on what is considered 'success'.

2) Level. The status of each collaborator (for example, the nature of their role, their power within their organization) is likely to impact on what is considered 'success'.

3) Phase. The importance of particular aspects of the collaboration will change over the course of the partnership.

4) Discipline. Scientific and non-scientific disciplines have different conventions and cultures, hence the background of each collaborator may shape their world view or perspective with regard to the importance placed upon certain methods and working practices, or how to derive outcome success. 
Through the case study presented in the remainder of this paper, we consider these concepts for collaboration under the lens of a novel academic-industry knowledge exchange partnership between $\mathrm{HCl}$ and the arts.

\section{An academic-industry collaboration case study: Exploring the ethics of VR through immersive theatre}

Our project was a 12-month collaboration between an $\mathrm{HCl}$ research group based at the University of Bristol, UK, and the immersive theatre group Kilter, based in Bath, UK. This collaboration was facilitated through a bidirectional knowledge exchange secondment, funded by the UK Engineering and Physical Sciences Research Council (EPSRC).

The $\mathrm{HCl}$ group comprised four employees - one lead researcher, two research associates and one administrator - embedded within the larger structure of the university's Department of Computer Science. Kilter, meanwhile, comprised two fulltime members of staff who owned and ran every aspect of the business. The primary objectives of the collaboration were:

1) Knowledge exchange. Specifically, for the $\mathrm{HCl}$ research team to better engage with young people on the topic of VR and its wider ethical issues by using novel methods derived from the dramatic arts, and for Kilter to develop their knowledge of university research processes, and for them to form a deep understanding of an emerging technology.

2) Public engagement. To engage diverse audiences through a series of workshops, culminating in an immersive performance event centring on the ethics of VR.

For the researchers, the collaboration with Kilter offered an opportunity to gain insight and upskill in the tools and techniques that are used by performance arts professionals to ideate and create narratives with and for mainstream audiences - to learn through involvement (Staley, 2017). Further, creating and performing a new piece of theatre (a design fiction) as the main project deliverable would increase the impact of their VR research within the local community, and potentially form the basis of future research.

For the Kilter team, the collaboration enabled direct and sustained access to expertise in an emergent technology of potentially high societal impact, to gain indepth knowledge and artistic inspiration, and to refine their art of simultaneously entertaining and educating audiences.

\section{The process: Participation with the VR designers and users of tomorrow}

\section{Early partnership building}

The first three months of the project focused almost exclusively upon interorganizational knowledge exchange. This consisted of a series of fortnightly meetings of two or three hours each that were hosted at the university. This phase entailed structural, procedural and cultural knowledge exchange. Both teams shared details about the methods undertaken in previous projects, particularly within collaborative endeavours.

At this stage, the bulk of the knowledge exchange flowed from the university team to Kilter. With little prior knowledge or experience of VR, considerable time was required to bring the Kilter team up to speed on the history, research landscape and current state of the art. During this time, the $\mathrm{HCl}$ team also facilitated practical 
experiences for Kilter to use and experiment with different VR systems and content, and to visit university labs within the Faculty of Engineering in order to understand more about the research currently being undertaken.

The relative complexity of the university as a partner, as well as the topic of the collaboration, required that the $\mathrm{HCl}$ team invest significant time in this early stage of the project to allow Kilter to ask questions and test their knowledge. While this process took time, it was considered crucial by both sides that a solid shared grounding in VR as it exists today be reached, upon which possible visions for its future could be explored. Learning was also supported using online channels, with the $\mathrm{HCl}$ researchers responding to Kilter queries via email between meetings, and the Kilter team documenting their own learning through an online blog.

\section{Workshop overview}

With limited time and resources available, it was decided relatively early on that the focus of the workshops would be on young people, on the basis that it would be this group that would be living with any long-term societal implications of VR, and that would be the custodians of future technology design. Moreover, from an $\mathrm{HCl}$ perspective, it is with young people that arts-based participatory techniques are often most impactful (for example, Dunn and Mellor, 2017). To achieve this, a series of participatory workshops were conducted with twelve Year 9 and 10 students (age range 13-15) from a secondary school in South West England as part of the school's extra-curricular enrichment programme. These workshops were to be co-developed by both teams, but they would ultimately be led by the Kilter team with support from two researchers from the $\mathrm{HCl}$ team.

Recognizing the likely attraction of virtual reality to the 'usual suspects' of young male video gamers, we worked closely with the host school to promote the creative aspects of the sessions in order to assemble a group of diverse interests and voices. Hence, the workshops involved students of mixed ages (within a two-year range), genders and academic interests.

\section{Challenges around access to schools}

Securing and organizing a series of workshops within a busy school during term time proved to be one of the most challenging aspects of the project. Both teams underestimated the time required to establish the partnership with our host school, falsely assuming that we could easily leverage an established network of young people based on our respective histories of working with such groups. By accessing the enrichment programme of our host school, we were able to side-step the need to find space within the general teaching curriculum. However, securing the workshops still took several weeks of negotiation.

The planning process also exposed differences in approach and viewpoint as to how the workshop sessions should be characterized and managed. The Kilter team felt most comfortable in an environment of spontaneity, and they were keen to take an ad hoc, flexible workshop approach - that is, not to be tied down to a rigid plan, and instead be free to work with and respond to the 'feeling in the room'. This contrasted with the $\mathrm{HCl}$ team, who saw the workshops as participatory research sessions that should follow a much more rigid structure of timed activities, where observations and other evaluative metrics could be carefully recorded. Compounding this, as an ultimately university-led venture, the researchers were obliged to explain clearly to their institution and the workshop participants exactly what would be happening 
and when in order obtain ethical clearance from the university. Without full ethical clearance, the workshops would not be permitted to proceed.

The requirement to obtain ethical approval from the university - a process that closely scrutinized the workshop activities, data collection, security provisions and safeguards for facilitators and young people alike - imposed a series of requirements upon participation that the Kilter team were not anticipating. Four issues were particularly salient, which we discuss in turn.

\section{The need for signed consent from young person and guardian}

All activities within the workshop would need to be explained as clearly as possible to students and guardians prior to the event to ensure that they were giving informed consent to their involvement. This placed a limit on the spontaneity of the session plans. However, some leeway was provided to the Kilter team by describing only the 'types' of activity that would be involved in the workshops, without necessarily tying each activity to a specific single workshop or time period.

\section{The requirement of up-to-date certification for working with under-18 year olds}

In the UK, all adults working with children and young people within a school environment must have up-to-date government clearance via a background criminal records check. Several team members did not have this clearance, and the lead time to obtain it can be several weeks. Fortunately, the host school were able to make a dedicated member of their staff available to supervise the sessions. This staff member did not actively participate in the workshop sessions (although they were invited to do so, if they so wished), and monitored the sessions quietly and unobtrusively from the back of the room.

\section{Data collection and storage}

University ethical protocol mandates that all data collected during research work must be stored within university-held storage structures by specific (named) university employees. Access to this information is subject to screening, anonymization and the explicit written permission of the people involved. This requirement created a slight tension between the researchers and the Kilter teams in terms of what constituted 'research data'. From the perspective of the researchers, all audio, video and textual materials created during the sessions were 'research data'. From Kilter's perspective, static 'snapshot' photographs taken by them in which faces could not be clearly seen, and materials written by the students that were not clearly attributed to them by name, were not 'research data', but simply supplementary materials that could, for example, be published online as a part of a blog detailing the workshops.

To manage this, the teams agreed to keep all materials confidential, and to seek explicit additional permission from the staff and students of the school before publishing any of the audio, visual or textual material generated during the workshops, either online or in print.

\section{Exposing young people to virtual reality technology safely within a classroom environment}

During one of our workshops, we provided the students with a short first-hand experience of virtual reality using commercially available VR headsets. For many of the students, this was their first experience of the technology, and for some it was 
a major draw for their participation in our workshops. At the time of writing, health and safety guidelines for virtual reality are relative sparse, and the effects of exposing younger adults and children to sustained sessions of VR remain unclear. However, the leading manufacturer of VR equipment recommends a minimum age of 13 (Oculus by Facebook, 2021).

From an ethical standpoint, we had to balance allowing the students to engage with the technology and maintaining a safe environment. This we achieved pragmatically by: (1) keeping their exposure to VR relatively short (five minutes); and (2) using a low-intensity VR experience by choosing an experience that was relatively benign and calm - a short immersive film of a coral reef.

In addition, students were supervised and remained seated throughout, and they were told to close their eyes and remove the headset immediately should they feel uncomfortable in any way. In practice, none of our workshop participants experienced any ill effects from their experience.

\section{School Workshop 1: Innovative methods and some differences in organizational ethics}

\section{Quickly breaking down hierarchies: The roundtable discussion}

The objectives of our first workshop were twofold: (1) to create an environment within which the students felt comfortable to speak and share (both with each other, and with the research team); and (2) to begin to understand how the students envisioned future societies and the role of VR technologies within them.

As a first activity, the Kilter team directed everybody (the students and the research team) to move from the tiered seating of the classroom to sit in a circle. The students were asked if they would be willing to move their chairs into a space in the centre of the room. The students were given autonomy over who they wished to sit next to, and the researchers also joined the young people in the circle. This would remain standard practice for the following workshops.

Each person was then asked to introduce themselves and generate a word describing their feelings about the future in order to introduce all parties to each other and to promote the notion that every participant had an equal voice. This immediately revealed a wide range of outlooks: optimists and pessimists, those curious and openminded about future society, and others with subversive ideas of their own. The use of the circular seating arrangement seemed to support a reduction of the sense of hierarchy within the classroom environment, and granted everyone a platform to be heard. For instance, after the students were asked to introduce themselves and share their feelings about the future, it often opened up a dialogue with other members in the circle (with other students, not just the research team), who made their own comments and asked questions about the ideas being shared.

\section{Introduction of methods for participant engagement}

Kilter also exhibited the benefits of balancing group-based and individual-based methods. Within the circle, the Kilter team shepherded initial ideas into a group discourse around the future of society through local, national and global lenses. They then anchored the discussion by producing some conversation starters in the form of written predictions about the future that they had brainstormed before the session, before introducing their first novel ideation approach - the 'washing line' method. The 'washing line' approach was essentially a linear timeline. During the discussions, whenever a new prediction about the future was suggested, the author of the idea 
was asked to write it down on a Post-it Note and pin it to a chronologically labelled line of string that traversed the breadth of the classroom. The students were then asked to move in physical space to stand at the place on the line that they felt best represented the year that they believed their prediction would become a reality. The activity provided a temporal way for the students to represent their ideas, mapping their predictions for world and personal events from the present day until the end of the century. This was not only an effective way of engaging the students to envision their ideas, but it also served as a visual representation of consensus and discord, which we were able to reflect upon and adjust as a team following the workshops. Moreover, the Kilter team was particularly interested in uncovering ideas that represented the near (and believable) future, to be used in the final production script.

A second technique deployed by Kilter was the use of workshop journals. Each student was provided with their own journal within which to develop creative narratives inspired by previous workshop content. Within their journals, students were repeatedly tasked to reflect upon specific questions and ideas about the future role of VR technologies that were posed during the workshops. From a research perspective, these journals served as an artefact for communicating the students' ideas about the design and ethics of VR in a future society - in a similar vein to developing a 'design fiction'. Yet, the individual and insular nature of this activity was also a great lesson in participant management: it enacted a rapid de-escalation in the intensity of the group interactions during prior activities. Moreover, it afforded an opportunity for the less vocal students to contribute without feeling the pressure of direct attention by the whole group.

To close the session, one member of the Kilter team wrote down the concepts raised during the session on a whiteboard, before inviting the students to direct him to write down the 'things that we discussed today'. The students shouted out concepts as he wrote them in real time. Following this, he asked the student group, 'What was the most important topic that was raised today?' As answers were expressed and opinions exchanged between the class members, he asked the students to use a show of hands in order to gauge the most impactful topics.

\section{Workshop 1 outcome summary: Laying down some early roots of inspiration}

Guiding the design of the final production, the activities of the first workshop identified several themes that appeared to be of particular concern to our cohort. These themes were developed further during debrief and planning meetings that were held between each workshop. Given the short time between the sessions, analysis of the previous workshop stopped short of a formal, academic qualitative method (such as thematic analysis). Instead, each member of the team brought to the debrief and planning meeting notes based on their experience of the previous workshop and any observation notes that they had taken during the session itself. Each member of the team shared what they believed to be the emerging themes from the workshop, with regard both to the workshop process and to the conceptual themes relevant to VR and ethics. A subset of themes that were noted by multiple members of the team were taken forward for further elaboration within the next workshop, and also as inspiration for the script of the final production.

Central among these was a perception that digital agents - for example, robots and artificial intelligence assistants such as Apple's Siri - were seen somehow as a de facto solution to various challenges for the planet, yet they also came with several implications that were not necessarily all positive. The students were most vocal at the prospect of such digital agents replacing real human-to-human relationships, 
and this seemed to divide opinion. Some students were unconvinced that this would happen in the near future, while others disagreed, stating that digital agents were already present and replacing human beings in many industries, such as the education industry. Opinions fragmented even further when the discussion turned to whether such agents would be positive or negative for society.

\section{School Workshop 2: Providing participants' value and handling competing organizational motives}

\section{Furthering participant learning}

The second workshop session began with the Kilter team leading a five-minute discussion reflecting on the previous session, with several of the students' journal ideas being shared as a basis for further ideation. Anonymity was provided to the students during this process; however, they were encouraged to take ownership of the idea if they wished. This activity served to anchor the session in the themes of the previous workshop (the ideas shared were those that had been highlighted during the debrief and planning meeting), as well as fostering group relationship building by allowing the students to build on each other's thoughts. The students were additionally given an opportunity to share any further thoughts that had not been covered if they wanted to, reinforcing the circle as a place where everyone had a voice. Yet, while the first workshop had helped us to understand how the students might visualize the future and the role of technology within it, we now wished to narrow the scope further towards the role of VR within this world.

To do this, part of the second workshop was dedicated to equipping the students with a depth of knowledge about VR and its history. By teaching them about the past, we hoped to equip them to better understand the present and to imagine the future. Moreover, given that we were working in a school context, we felt it was important that we contribute to student learning in a variety of different methods - experiential and theoretical -in addition to the creative activities from the previous session. Kilter and the research team had originally conceived the delivery of this educational content about VR as a more constructivist activity, with a great deal of student self-determination and socially constructed learning, in line with the other content delivered in the workshops. However, two external pressures impacted our ability to do this. First, there was an extremely limited amount of time available within the workshop to allow for a full and socially constructed method for the students to learn about such a rich set of technical topics within 30 minutes. Second, the school and a number of the attending students interested in STEM subjects intimated a desire to hear the researchers involved talk about their work, and to share their expertise in VR.

Hence, following the recap discussion, a member of the $\mathrm{HCl}$ team provided the students with a 30-minute talk about the roots and emergence of VR, as well as about some notable projects that the researchers had undertaken in this space. To allow use of a projector to provide additional visual materials, this was presented with the students sitting in tiered rows, with the content delivered through a combination of visual slides and spoken content. Immediately following the talk, the students were provided with VR headsets to experience a short immersive film of swimming in a coral reef. The objective was to ensure that every student in the class had a baseline of at least one experience of using VR, which they could use as a launchpad for their imaginative design.

For many of the students, this was their first experience of VR. The opportunity to try it out allowed them to cement their understanding of the VR concepts that had 
been presented through practical experience. Although many of these students had experience of immersive virtual environments through video games, it was the first time that they had been fully encapsulated within a 360-degree environment of this kind. Nevertheless, this did not seem to obviously affect their enthusiasm, as several students from both sides of the STEM/arts subjects divide stayed behind for a few minutes after the session to ask the researchers questions about the technology.

\section{Balancing research and creative goals}

Providing an actual VR experience was clearly impactful for the students, yet it also highlighted some differences in prioritization for the $\mathrm{HCl}$ and Kilter teams. As there were only six VR headsets available for concurrent use (and only two researchers trained in their administration), tight time management and orchestration were required to keep students engaged while waiting for their turn. To ensure fairness, students selected among themselves the order in which they would take part in the VR experience. Meanwhile, the Kilter team ran a concurrent task where students who were waiting for their turn elaborated upon the future societies described in their journals in order to include a role for VR. While this kept the students engaged, the activities had different goals and were weighted with different importance for the $\mathrm{HCl}$ and Kilter teams.

For Kilter, the journal activity was an important part of expanding upon the students' narrative ideation, providing them with more material to inspire the final immersive theatre performance around the ethics of VR. The $\mathrm{HCl}$ team felt that the opportunity to provide the young people with practical experience of VR, which was a selling point of the workshops to the school, and a strong basis of knowledge was equally important. They believed that this was required in order to scope students' design in the not-too-distant technological future - which could support the students to deliver plausible ideas for future VR technologies and generate the most impactful content for the final production.

In practice, completing both tasks running in parallel placed severe pressure upon the limited time available in the session. Due to the set-up time required, each five-minute VR experience for a group of six students took ten or fifteen minutes to complete, causing an overrun. To complete both tasks, additional time would need to be carved out of the following session to enable the remaining students to undertake their VR experience. This additional logistical pressure was a source of frustration for Kilter, who had been very careful about the format and chaining of activities in order to manage the energy of the students. However, they were understanding, and all parties agreed that any future researcher-led methods in the workshops would be better codesigned with Kilter.

\section{Workshop 2 outcome summary: Providing a practical reference point for virtual reality}

Given the emphasis on providing the participants with a grounding in the history and direction of VR technologies during Workshop 2, there were limitations on establishing themes for use in the final production. Although Kilter had deployed their journal activity, relatively little time had been available for any firm narrative ideation. Hence, the next debrief and planning meeting focused on understanding the students' perception of their VR experience, and thinking about how this could be used to inform further creative ideation activities.

All of the students had expressed positive feelings about their VR experience, but with seemingly little immediate concern about the potential ethical implications of 
the technology (at least in its current form). On first glance, it did not appear that VR caused the same trepidation for them as other technologies (such as digital agents) mentioned in the first workshop. Given the brief and somewhat benign VR experience that we offered, this was not entirely surprising. Nevertheless, one particular idea began to take shape - the use of VR to allow people to experience places and activities that they could not experience in real life - and this was taken through to the final workshop session.

\section{School Workshop 3: Applying our knowledge of virtual reality ethics Describing the role-play ideation method}

Our final workshop built upon the students' first-hand experience of using VR from the previous session, and reoriented their focus towards considering what (if any) ethical implications they felt it raised. The goal of the session was for the students to ideate their own application for VR, before using peer review and reflection to identify any ethical issues surrounding their application.

The students began by undertaking a refresher discussion about their VR experience in the circle, as the subgroup of students who were yet to undertake their VR experience were given the opportunity to do so. After the subgroup had finished their VR experience, they were invited back into the circle, where they were given a range of topics representing different societal lenses on A4 sheets of paper, which they would use as a basis for their initial VR design ideas. These topics had been curated by Kilter during previous projects, and included law and order, the environment, health care and history. The participants formed small groups depending on their area of interest, and they were asked to brainstorm their idea for a VR application, and then to elaborate upon their idea in a brief presentation to the rest of the group.

To facilitate their presentation, the Kilter team employed role play, with one member adopting the persona of a news anchor, and the other playing a 'roving reporter' who was presenting a news story about a VR start-up convention from the studio, before visiting 'tech start-ups' (that is, the student teams) 'on the ground'. The 'reporter' interviewed each team in turn, guiding the students towards explaining their ideas with semi-structured questions. Noting the increasing tendency towards student-to-student interaction during the role play, the Kilter team later extended the activity further. They took votes from all the students on their favourite idea, with the teams that had conceived the two leading ideas then invited to take part in a 'press conference'. Here, they fielded questions from the other students, who took on the role of journalists. The goal was to allow for a more student-led, expanded ideation of the created concepts and any ethical concerns.

\section{Using role play to engage}

As professional dramatists, the Kilter team were skilled at adopting convincing personas quickly and creating an entertaining narrative that would have been difficult for the researchers to have undertaken without considerable training and practice. The researchers observed the ability of the Kilter team to focus attention, provoke discussion and evoke audible and visible emotion from the students during the role play. For instance, the role play was accompanied by audible laughter from many of the students, while individual students began to mirror the Kilter team by appearing to adopt their own characters or amplified personas of themselves. Furthermore, students began engaging each other while in character, and attempted to break into role-play interactions by making comments and addressing the Kilter team from across the room. 
Presenting ideas in the classroom in front of their peers can sometimes be intimidating for young people. This can be caused by a lack of confidence or self-efficacy with regard to the topic or idea being discussed, the social consequences of being incorrect and being subject to mockery, and personality and cultural factors, as well as teaching-centred learning environments and intimidating power imbalances between students and their teachers (Zhouyuan, 2016). Hence, Kilter's use of spontaneous role play attempted to make the process informal, inclusive, engaging, and perceived as low risk for the students to actively participate. Key to this was the role of the reporter, who - as part of 'the act' - structured and supported the students' explanations of their idea. This was designed to support students' confidence, and to help them to improvise or play a role of their own. At the same time, the 'press conference' format allowed ideas to emerge quickly and to be explored in further depth for feasibility, without alienating or discouraging other participants by giving them a role to play. Through playing a role, students were granted deniability for any ideas that may have missed the mark - that is, such ideas could be attributed to the character, rather than the individual - something from which many 'shy' actors have benefited (Scott, 2017).

\section{Workshop 3 outcome summary: New applications for virtual reality, and a greater understanding of the ethics of virtual reality technology}

The role-play activity within Workshop 3 created a diverse range of ideas for future VR applications. These included the use of VR headsets to control environmentally friendly robots, to relive historical events for educational purposes, to simulate the physical and judicial consequences of carrying knives, and to extend the lives of terminally ill people. The students cast an even number of votes for the latter two ideas, with the press conference generating several ethical concerns. Notable among these was the risk that a VR experience of knife crime could be 'triggering' for those experiencing it - both for those experiencing these events for the first time within virtual reality, and for those who had been previously directly or indirectly involved with knife crime in real life.

The second idea, 'Bucketlist VR', described a VR-based system for extending the lives of terminally ill people. This became a major influence upon Kilter's final production. Bucketlist VR was conceived as a broadly positive vision of a VR company that enabled terminally ill hospital patients to live their lives beyond their physical confines, doing anything that they wished to do and being able to spend more time with family members in a virtually constructed alternative environment to a hospital. However, the idea also raised a number of ethical issues for the students, including the notion of people preferring to live their lives in VR rather than their actual reality, and whether such an application would negatively (or indeed positively) affect the process of grief and grieving.

\section{Final Output: VR100 - we warmly invite you to Grandad's 100th birthday party}

The final part of the collaboration was Kilter's creation of a bespoke dramatic performance VR100. The purpose of VR100 was to develop some of the more salient themes that emerged from the workshops into a piece of dramatic design fiction that we could use to spark discussion and reflection on the ethical dimensions of VR with a public audience. Through review of the workshop discussions, it was agreed between the two teams that the concept of VR100 would combine three core ideas that had been generated by the students during the three workshops: (1) the prospect of using 
VR to replace human-human interactions with those involving virtual/digital agents; (2) the use of VR within the context of death and mortality; and (3) the potential use of VR as a means of experiencing new things, visiting people and places. It was decided that the script would be set in 2079 - a year that the students considered to be realistic for the technologies being discussed, identified using the 'washing line' technique from the first workshop. The creative team at Kilter prepared the initial script for the performance independently of the $\mathrm{HCl}$ team, after which it was reviewed and edited by both teams over the course of several weeks.

The story of VR100 centres on the relationship between a granddaughter and her late grandfather, whom she has attempted to recreate post-mortem through the services of virtual reality company 'BucketList'. The central ruse of the performance is that the audience is led to believe that they have been invited to celebrate the grandfather's 100th birthday party, when in fact he has already passed. As the story unfolds, clues as to the true whereabouts of the grandfather are slowly revealed through the interaction of the granddaughter and the CEO of BucketList, who is also present at the party. Hence, after singing 'Happy Birthday', the audience was to be invited to 'visit' the grandfather in his new virtual home, 'exactly how they remembered him'.

However, an argument abruptly ensues between the granddaughter and the CEO, after the latter accidentally exposes a secret about the grandfather that had emerged during the process of researching his life. Upon hearing this, the granddaughter interprets her grandfather's behaviour as a betrayal, and demands that the CEO 'pull the plug' on the recreation. At this point, the story ends.

The performance of VR100 took place as a free public event in the function room of a local public house, with the lead characters played by the Kilter team. To set the scene, the room was decorated appropriately with balloons, party-poppers, drinks and snacks. As the audience filed into the room, they were greeted warmly by the actors, who were already in character as the granddaughter and the BucketList CEO.

The audience were recruited through an online advertisement circulated via the social media accounts of the university and Kilter. So as not to reveal the ruse at the core of the performance, the advertisement made no mention of death or mortality, and only light reference to immersive technology: 'Grandpa has reached 100! You are cordially invited to raise a glass to toast his first century ... \& launch his next.' The event itself was described as: 'a short performance piece that we hope will provoke interesting conversation around the ethics of immersive technology. By attending this event, you will be contributing to cutting-edge research about the future uses and implications of immersive technology.' It was hoped that this approach would encourage participation from a mixed audience, including technology enthusiasts, general theatre-goers and the simply curious. All the students and teachers from our workshops were directly invited, and several were able to attend.

Immediately following the 25-minute performance, the audience were invited to engage with the team in an open $Q \& A$ session to discuss the issues raised by the story and to highlight any ethical issues that the audience felt were important.

At least initially, the audience members grounded their questions in examples from the performance itself. Most agreed that the use of VR for realizing dreams and desires was something they could imagine, and that it could be very valuable (for example, for the elderly or infirm). However, several highlighted that the recreation of real people within VR was ethically challenging. There were concerns about whether the digital recreation of themselves or others might be used for manipulation, and some suggested that it might impinge upon their human rights and the control of personal data. Others were concerned about whether it might exploit, interrupt or even circumvent natural grieving processes. 
As a piece of design fiction, the VR100 performance succeeded in its goal of provoking an interesting and wide-ranging conversation about ethics, and the overwhelming anecdotal response from our audience (including several students from our workshops) was that the experience was interesting and enjoyable. From a research perspective, the Q\&A session revealed that the ethical touchpoints of immersive technology were much wider than the researchers had themselves originally imagined. While the conversation initially focused on VR, it soon broadened to encompass and find links between a range of contemporary (and often dystopian) technological issues, including aspects of sex and relationships, technophobia, digital inclusivity and transhumanism.

However, while this broad conversation was fascinating, it was difficult to keep the conversation focused on $\mathrm{VR}$, and the conversation regularly dwelt on dystopian rather than utopian ideas. While it might well be the case that the audience truly felt that the negatives were more salient than the positives (we have little evidence one way or the other), we must also recognize that, in its use of dramatic licence to flip the concept of mortality on its head (the original concept was to provide VR experiences to people who were very much alive), the script of VR100 was provocative. While this certainly helped to set up a central ruse and generate dramatic impact, from the researchers' perspective, there were consequences of bringing into play the ethics of a range of surrounding technologies and socio-technical issues that are very familiar in contemporary dystopian science fiction (notably the Netflix series Black Mirror, which was popular at the time, and which was mentioned by several audience members). Part of the appeal of dystopian science fiction lies in its ability to provide a safe space within which to explore our fears. When exploring near-future technology in a dramatic context, it is difficult to find a balance between dystopian or utopian world views while generating engaging and dramatic storytelling. Nonetheless, researchers using design fiction in this way should be very mindful that the conversation can become rapidly detached from a core area of interest, and/or focus only on negative issues, if a narrative strays too close to dystopian science fiction.

\section{Discussion: Impact and insight}

\section{Assessing impact for ongoing research and arts-based $\mathrm{HCl}$ methods}

As described by Senior et al. (2016: 27), 'In pushing models of knowledge exchange towards an understanding of collaboration as co-creation, the value of collaborative work extends far beyond the mere exchange of knowledge between sectors.' For the $\mathrm{HCl}$ team, then, the present collaboration has been impactful across three lines: (1) informing ongoing research projects about VR technologies; (2) advancing methodological competencies; and (3) diversifying outreach efforts. The insights gathered from the engagement with young people and the audience of the VR100 performance have raised several interesting points for discussion in the team's current thinking about the adoption of VR technologies in the home, as well as unearthing fertile ground for further research and engendering the formulation of additional research questions directly related to some of the themes raised in VR100.

The collaboration also had a methodological impact on the $\mathrm{HCl}$ team and their perceptions of artefact-based participatory design. Within the workshops, the use of role play embedded with compelling narratives and theatrical elements was observed to quickly generate a range of valuable ideas from participants. The researchers also learned participant management skills and workshop structuring techniques - including the timing of activities to manage and manipulate participants' energy. While Kilter's 
theatrical training may have been a key ingredient in the success of these methods, being able to observe them over a period of several hours, as well as to help plan the workshop activities, provided the researchers with a blueprint, and the confidence to deploy these methods for themselves.

One area where Kilter's methods have aligned particularly well is with the $\mathrm{HCl}$ method of design fiction - developing diegetic prototypes as a provocative topic of discussion. The VR100 performance, for instance, may constitute a high-fidelity alternative to other diegetic prototype media, as it portrays a functional piece of technology within a fictional world, supporting dialogues, plot rationalizations, character interactions and narrative structures (Kirby, 2010). Our future work will consider how VR100 could be expanded to do more to help encourage audiences to suspend their disbelief by introducing complementary artefacts, such as digital mockups of the VR technology that was talked about, but never actually shown, during the performance.

\section{Insights for effective university-industry collaboration: $\mathrm{HCl}$ and the creative arts}

As previously discussed, recent research articles have posited insights and best practices for general university-industry collaboration. However, we also recognize that variance in structure, size and culture of different academic and industry teams places limitations on their generalizability (Rybnicek and Königsgruber, 2019). Hence, we present five nuanced insights from our collaboration which we feel are particularly relevant for university-based $\mathrm{HCl}$ researchers who wish to partner with creative arts organizations. Several of these insights may complement existing findings of universityindustry collaboration in other domains.

\section{Understanding structural and procedural norms can be addressed through dialogue, but bridging cultural differences may require organizational immersion}

Taking time to gain an understanding of each partnering organization's working practices is requisite to the success of academic-creative industry collaborations, but this process took a great deal more time than we anticipated. Part of this was a result of the difference in size of the organizations, and the way in which time and resources are accessed. Kilter, for example, is a small-medium enterprise with a flat power hierarchy (Boardman and Bozeman, 2015). While many university-based $\mathrm{HCl}$ teams ostensibly hold a similar power structure (small groups of people of equal rank who are brought together from various areas of study, such as computer science), they are often subsumed by more complex hierarchies on which they must rely for key activities (such as resource procurement, ethics, finance and communication channels) (Schofield, 2013).

Consequently, within our collaboration, although the $\mathrm{HCl}$ team held a similar degree of autonomy to Kilter over the planning of collaboration activities, the implementation of these plans could not be undertaken with as much flexibility - not least due to a requirement for clearance by the university ethics committee for all work undertaken by its researchers. Echoing Rybnicek and Königsgruber's (2019) model, we therefore suggest that it is vital that time is taken prior to beginning a universityindustry partnership to understand each organization's structure, as well as their logical, procedural and ethical rules and regulations. Reflecting upon our collaboration, a great deal of time early on was spent bringing Kilter up to speed on wider university structures and procedures in order to set realistic expectations, particularly with regard to university requirements around research ethics and data storage. 
While this exchange of knowledge helped to bridge structural and procedural differences, fundamental diversions in organizational culture were harder to unroot. In particular, finding a balance between Kilter's desire for spontaneity - to be able to respond quickly to the 'mood of the room' - and the $\mathrm{HCl}$ team's desire to maintain a controlled analytical approach created friction between the $\mathrm{HCl}$ and Kilter teams. The ability to engage in spontaneous improvisation during workshop sessions was seen as a useful tool by Kilter to engage audiences, but, while the researchers could also see the potential value of this, they were anxious about the degree to which such unplanned activity impacted the informed consent of the participants.

With hindsight, and with more time, these frictions might have been eased with early, focused immersion in each other's organizations. Ideally, both partners should be afforded first-hand experience of the other's working practices and norms. During our collaboration, for example, all meetings were held at the university, and far too little time was afforded for building trust and experience around what 'spontaneous' activities actually entailed in practice.

\section{Mutual openness to change can foster shared culture, but adherence to rigid research ethical procedures should be accepted early on as a university 'red line'}

The requirement for a rigorous and accountable ethical framework is culturally, procedurally and structurally inexpugnable for $\mathrm{HCl}$ researchers whose work involves members of the public. It is also critical, but sometimes forgotten, that ethical review by the host university serves to protect not only the participants, but also the researchers themselves. For the uninitiated, however, the rigour of the process involved in obtaining ethical clearance for researchers to conduct work outside a university can sometimes feel overzealous, if it is not sufficiently well explained. Hence, the importance of early planning for ethical review, and a realistic shared understanding of the time required for the process to be completed, should be communicated as early as possible.

In retrospect, a mistake was made in not involving Kilter more directly in the process of obtaining ethical clearance to conduct the workshops. As a result, the goals of ethical approval became somewhat abstracted, with confusion over what was and was not 'research', and what could and could not be done within the workshop sessions. This could easily have been avoided.

\section{Collaborators should aim to progress from being multidisciplinary to interdisciplinary, but should also expect this process to be asymmetrical}

Knowledge exchange partnerships are frequently a multidisciplinary endeavour, with experts from different fields approaching a shared problem through different lenses. As described in previous literature, frequent communication through a variety of channels (for example, face to face, through demonstrations and by email) is the basis for developing mutual understanding and common language (Hong et al., 2010; Lee, 2011; Rybnicek and Königsgruber, 2019).

In our collaboration, we found the process of knowledge exchange to be somewhat asymmetrical. Particularly in the early stages of the collaboration, knowledge exchange between the teams did not progress in a collective, linear trajectory. There were periods of the project where the balance of learning tipped towards one party, before later tipping back. For instance, our early knowledge exchange leaned heavily on the academic side of the collaboration to educate Kilter about VR, only later switching to Kilter upskilling the $\mathrm{HCl}$ team in immersive theatre when planning for the participatory workshops began. 
Project leadership followed a similar pattern, with each team leading at different project stages and on different issues. Despite this, the gap in both expertise and leadership responsibilities soon began to narrow, and, as the collaboration evolved, it opened uncharted challenges and opportunities which necessitated a truly interdisciplinary approach. For example, the script 'read through' meeting for the VR100 performance became a setting for academic debate about which is the most prominent ethical issue of VR, as well as how it could be portrayed within the production, with opinions voiced on all issues by both Kilter and $\mathrm{HCl}$ teams. In this way, both knowledge and responsibility became shared over time. Thus, we posit that in $\mathrm{HCl}$ collaborations, partners should attempt to become more interdisciplinary developing expertise in each other's fields - rather than multidisciplinary - only bringing their own expertise. Interdisciplinarity is key to fostering innovation (Fontana and Corsatea, 2013) - the consideration of phenomena from new perspectives, or by using new tools to study them.

\section{Even with maximum transparency, different stakeholder objectives can unexpectedly conflict}

Rybnicek and Königsgruber (2019) highlight that collaborations in which individual (or groups of) team members intentionally prioritize their own agendas can lead to tension and a lack of trust. To mitigate this in our collaboration, time was taken to openly discuss and document individual, team and shared objectives prior to the project application to ascertain how the project would be mutually beneficial. Despite this, however, some differences of perspective and external pressures were not immediately identified, and were hard to completely eliminate once the collaboration was under way.

For example, both teams shared the desire to raise public awareness regarding the ethics of VR. However, opinions on the means by which public awareness might be measured, and what constituted 'value' and 'success' in terms of project outputs, differed. For the most part, these differences in opinion coexisted without incident, although they sometimes and unexpectedly came into direct competition. A salient example arose during the workshops, when Kilter saw greater value in being able to undertake a more flexible approach that allowed for spontaneous improvisation, whereas the $\mathrm{HCl}$ researchers wanted to stick to a predefined schedule of activities that could be validated against ethical commitments and modelled in order to document the process in academic literature. As described previously, compromise needed to be made, and joint planning sessions before each workshop became an important forum to allow each team to speak candidly about their concerns, and to come to an agreed plan.

Within our collaboration, none of these conflicts were sufficiently severe as to derail the process of completing the necessary work. However, resolving them required additional meetings, which consumed valuable project time and relied heavily on the goodwill of both parties. Hence, we advocate for honest, transparent dialogue early in the project to establish where project objectives and measures of success converge and separate (Barnes et al., 2002). Collaborators should also consider putting in place a protocol to handle any unforeseen conflicts of interest, and being prepared to negotiate suitable compromises - for example: who is in charge of negotiations for each collaborator, and who else is involved, and what is the venue for negotiations?

What happens if collaborators cannot come to an agreement? Which factors (for example, financial or ethical) should be considered or prioritized during compromise? Most importantly, collaborators should describe and clearly define their own visions for achieving shared project goals (the inputs, methods and outputs) at the earliest convenience. 


\section{Developing a shared language can be complex and littered with assumptions}

The development of shared language can help to bridge culture gaps between academia and industry, and also between individual disciplines (Canhoto et al., 2016). However, this process is not always straightforward, and assumed meanings can and do persist. As a consequence of the long-standing influence of the arts upon $\mathrm{HCl}$, there was considerable overlap already with the terminology used by the Kilter team. However, nuanced differences in specific definitions were magnified in practice. A salient example of this in our collaboration involved both teams' commitment to following a process of 'co-design' with the participants in our workshops. For Kilter, 'co-design' meant supporting the workshop participants to think about VR and its implications in a socially constructed way, with an emphasis on helping them to garner skills in creative narrative development and role play. Ultimately, however, Kilter would retain full control and ownership of the output of this exercise - the script of the VR100 performance - which would be written by them, but which would be 'inspired' by the workshop participants. For the researchers, however, 'co-design' was assumed to be analogous to 'participatory design' - a process that involved a much stronger emphasis on participant empowerment, shared ownership and continuous engagement in order to bolster the learning value of participatory processes. The $\mathrm{HCl}$ designers had imagined the workshop participants taking a role somewhere between 'design partners' and 'design informants' (Druin, 2002), empowered to share opinions, to give feedback, and to make key decisions within both the workshops and the final production process, which could be validated and supported by the $\mathrm{HCl}$ and Kilter teams. Literature suggests that such roles offer young people much greater opportunity to experience autonomy and empowerment, as opposed to feedback-only roles akin to 'testers' (Druin, 2002). In allowing this assumption to persist untested, the $\mathrm{HCl}$ team had envisaged the young people taking greater ownership of the process, for example, by directly helping to write the script, and perhaps by acting in the final production. In retrospect, a shared definition of co-design should have been more clearly established before the workshops took place.

\section{Conclusion}

Knowledge exchange collaboration between universities and the creative industry affords those involved an opportunity to progress from a multidisciplinary group of individuals to a more cohesive unit with shared knowledge, language and objectives. From the case study described here, a group of $\mathrm{HCl}$ researchers have expanded their knowledge of arts-based techniques for participant engagement, which they can carry forward to new projects and propagate within the $\mathrm{HCl}$ community. Meanwhile, the collaboration has helped the creative industry partner to garner a wealth of new knowledge within STEM disciplines, which they can build upon as a continuing source of inspiration in their own work.

Furthermore, the collaboration also offered each party an opportunity to reflect upon their own disciplines, language and wider organizational cultures. For the $\mathrm{HCl}$ researchers, partnering with professional artists highlighted the value of experimenting and developing innovative methods, and exposed the limitations of relying upon the status quo of well-established research techniques. For the creative partner, the experience of conducting ethically sound participatory research within the limits of a university-led venture has inspired them to embrace co-design more deeply and more broadly with their audiences than they had done previously. 
However, these outcomes were all contingent upon all collaborators being willing and able to constructively work through the inevitable challenges that can arise during university-industry collaboration. Every partnership will have its own nuanced areas of friction. However, we hope that the insights raised in this case study provide a basis for helping similar projects anticipate and mitigate their own challenges.

\section{Acknowledgements}

We would like to thank our design partners - the students and staff from Redland Green School in Bristol, UK. We would also like to thank the EPSRC for funding the project, as well as Ellie Cripps from the University of Bristol's public engagement team.

\section{Notes on the contributors}

Stuart Gray is a research associate in the field of human-computer interaction in the Department of Computer Science, University of Bristol, UK. Stuart's research focuses on the development and evaluation of technologies for health and well-being and child-computer interaction. More recently, Stuart's research interests have broadened to consider the social and ethical implications of emerging technologies.

Chris Bevan is a research associate in the field of human-computer interaction in the Department of Computer Science, University of Bristol, UK. Chris's research encompasses virtual reality, mobile/pervasive computing, social robotics and cybersecurity. Chris works on large cross-disciplinary and multi-institutional research projects with partners from academia, industry and the creative sector.

Kirsten Cater is the Associate Dean for the Faculty of Engineering responsible for Programmes and Delivery for the University of Bristol's Temple Quarter Campus, UK. Kirsten is the founder and Director of the Centre for Innovation and Entrepreneurship at the University of Bristol. Alongside these roles, Kirsten is actively involved in humancomputer interaction research, with interests including virtual reality, gamification, and technologies for physical and social well-being.

Jo Gildersleve is a research coordinator in the Faculty of Engineering, University of Bristol, UK. Jo focuses on the coordination of interdisciplinary research projects across computer science, as well as civil and water engineering. With her additional research interests in history, a subject in which she holds an MPhil, Jo is currently involved in a project that models stories from the classics of Ancient Greece within educational VR environments.

Caroline Garland is the Co-Director of the socially engaged arts company Kilter Theatre, based in Bath, UK. Under Caroline's leadership, Kilter have collaborated with a diverse range of partners to devise original theatre-based interventions that engage new audiences in the ethical environmental issues of the future. Previous collaborations include Invincible, a project undertaken with scientists at the University of Bristol, which developed theatre performances with the goal of provoking debate and scrutiny of synthetic biology.

Oliver Langdon is the Artistic Director of socially engaged arts company Kilter Theatre, based in Bath, UK. Kilter collaborates with a diverse range of partners to devise original 
theatre-based interventions that engage new audiences in the ethical environmental issues of the future. Oliver's artistic performances with Kilter have garnered a national reputation for involving audiences, igniting imaginations, encouraging debate and kick-starting positive change locally and globally.

\section{References}

Ames, M.G., Brown, B., Fels, S.S., Lindtner, S., Rosner, D.K. and Vertegaal, R. (2015). '10 years of alt. chi: Reflections and outlook'. In Proceedings of the 33rd Annual ACM Conference Extended Abstracts on Human Factors in Computing Systems. New York: Association for Computing Machinery, 839. https://doi.org/10.1145/2702613.2736010.

Arvanitis, S., Kubli, U. and Woerter, M. (2008) 'University-industry knowledge and technology transfer in Switzerland: What university scientists think about co-operation with private enterprises'. Research Policy, 37 (10), 1865-83. https://doi.org/10.1016/j.respol.2008.07.005.

Attia, A.M. (2015) 'National innovation systems in developing countries: Barriers to universityindustry collaboration in Egypt'. International Journal of Technology Management \& Sustainable Development, 14 (2), 113-24. https://doi.org/10.1386/tmsd.14.2.113_1.

Barnes, T., Pashby, I. and Gibbons, A. (2002) 'Effective university-industry interaction: A multicase evaluation of collaborative R\&D projects'. European Management Journal, 20 (3), 272-85. https://doi.org/10.1016/S0263-2373(02)00044-0.

Berbegal-Mirabent, J., García, J.L.S. and Ribeiro-Soriano, D.E. (2015) 'University-industry partnerships for the provision of R\&D services'. Journal of Business Research, 68 (7), 1407-13. https://doi.org/10.1016/j.jbusres.2015.01.023.

Blackwell, A.F. (2015) 'HCl as an inter-discipline'. In Proceedings of the 33rd Annual ACM Conference Extended Abstracts on Human Factors in Computing Systems. New York: Association for Computing Machinery, 503-16. https://doi.org/10.1145/2702613.2732505.

Bleecker, J. (2009) Design Fiction: A short essay on design, science, fact and fiction. Near Future Laboratory. Accessed 3 June 2021. http://drbfw5wfjlxon.cloudfront.net/writing/DesignFiction_ WebEdition.pdf.

Boardman, C. and Bozeman, B. (2015) 'Academic faculty as intellectual property in universityindustry research alliances'. Economics of Innovation and New Technology, 24 (5), 403-20. https://doi.org/10.1080/10438599.2014.988499.

Bstieler, L., Hemmert, M. and Barczak, G. (2017) 'The changing bases of mutual trust formation in inter-organizational relationships: A dyadic study of university-industry research collaborations'. Journal of Business Research, 74, 47-54. https://doi.org/10.1016/j.jbusres.2017.01.006.

Canhoto, A.I., Quinton, S., Jackson, P. and Dibb, S. (2016) 'The co-production of value in digital, university-industry R\&D collaborative projects'. Industrial Marketing Management, 56, 86-96. https://doi.org/10.1016/j.indmarman.2016.03.010.

Chilana, P.K., Ko, A.J. and Wobbrock, J. (2015) 'From user-centered to adoption-centered design: A case study of an $\mathrm{HCl}$ research innovation becoming a product'. In Proceedings of the 33rd Annual ACM Conference on Human Factors in Computing Systems. New York: Association for Computing Machinery, 1749-58.

Coulton, P., Lindley, J.G., Sturdee, M. and Stead, M. (2017) 'Design fiction as world building'. In Proceedings of the Third Biennial Research Through Design Conference 2017, 163-79. https://doi.org/10.6084/m9.figshare.4746964.

Crawley, E., Hegarty, J., Edström, K. and Garcia Sanchez, J.C. (2020) Universities as Engines of Economic Development: Making knowledge exchange work. Cham: Springer International.

Druin, A. (2002) 'The role of children in the design of new technology'. Behaviour and Information Technology, 21 (1), 1-25. https://doi.org/10.1080/01449290110108659.

Duarte, E.F. and Baranauskas, M.C.C. (2018) 'Revisiting interactive art from an interaction design perspective: Opening a research agenda'. In Proceedings of the 17th Brazilian Symposium on Human Factors in Computing Systems. New York: Association for Computing Machinery, 1-10. https://doi.org/10.1145/3274192.3274227.

Dunn, V. and Mellor, T. (2017) 'Creative, participatory projects with young people: Reflections over five years'. Research for All, 1 (2), 284-99. https://doi.org/10.18546/RFA.01.2.05.

Edmonds, E. (2017) 'The art of interaction: What $\mathrm{HCl}$ can learn from interactive art'. Synthesis Lectures on Human-Centered Informatics, 11 (1), 1-73. https://doi.org/10.2200/ S00825ED1V01Y201802HCI039.

Ehn, P. (1988) 'Work-Oriented Design of Computer Artifacts'. PhD thesis, Umeå University. 
ESRC (Economic and Social Research Council) (2021) 'Tips for doing knowledge exchange'. Accessed 18 August 2021. https://esrc.ukri.org/research/impact-toolkit/tips-for-doingknowledge-exchange/.

Etzkowitz, H. and Leydesdorff, L. (2000) 'The dynamics of innovation: From national systems and "mode 2" to a triple helix of university-industry-government relations'. Research Policy, 29 (2), 109-23. https://doi.org/10.1016/S0048-7333(99)00055-4.

Fitzpatrick, G. (2018) 'A short history of human computer interaction: A people-centred perspective'. In Proceedings of the 2018 ACM SIGUCCS Annual Conference, 1-3. https://doi.org/10.1145/3235715.3242569.

Fontana, M. and Corsatea, T.D. (2013) 'Empirical evidence of innovation in economic theory: Scientific interaction and knowledge diffusion at the Santa Fe Institute'. Complexity Economics: Complexity, crises \& choices, 1 (2), 77-105

Goduscheit, R.C. and Knudsen, M.P. (2015) 'How barriers to collaboration prevent progress in demand for knowledge: A dyadic study of small and medium-sized firms, research and technology organizations and universities'. Creativity and Innovation Management, 24 (1), 29-54. https://doi.org/10.1111/caim.12101.

Green, D.P., Schofield, G.P., Hodge, J., Rose, M., Cater, K., Bevan, C. and Gray, S.I. (2019) 'Using design fiction to explore the ethics of VR "in the wild"'. In Proceedings of the 2019 ACM International Conference on Interactive Experiences for TV and Online Video, 293-9. https://doi.org/10.1145/3317697.3323346.

Guan, J.C., Yam, R.C. and Mok, C.K. (2005) 'Collaboration between industry and research institutes/ universities on industrial innovation in Beijing, China'. Technology Analysis \& Strategic Management, 17 (3), 339-53. https://doi.org/10.1080/09537320500211466.

Hadjimanolis, A. (2006) 'A case study of SME-university research collaboration in the context of a small peripheral country (Cyprus)'. International Journal of Innovation Management, 10 (1), 65-88. https://doi.org/10.1142/S1363919606001405.

Henderson, J., McAdam, R. and Leonard, D. (2006) 'Reflecting on a TQM-based university/industry partnership: Contributions to research methodology and organisational learning'. Management Decision, 44 (10), 1422-40. https://doi.org/10.1108/00251740610715731.

Hong, J., Heikkinen, J. and Blomqvist, K. (2010) 'Culture and knowledge co-creation in R\&D collaboration between MNCs and Chinese universities'. Knowledge and Process Management, 17 (2), 62-73. https://doi.org/10.1002/kpm.342.

Indarti, N. and Wahid, F. (2013) 'How do Indonesian industries perceive university-industry collaboration? Motivations, benefits and problems'. International Journal of Technology Transfer and Commercialisation, 12 (1-3), 157-71. https://doi.org/10.1504/IJTTC.2013.064169.

Jeon, M., Fiebrink, R., Edmonds, E.A. and Herath, D. (2019) 'From rituals to magic: Interactive art and $\mathrm{HCl}$ of the past, present, and future'. International Journal of Human-Computer Studies, 131, 108-19. https://doi.org/10.1016/j.ijhcs.2019.06.005.

Johnson, M.T. (2020) 'The knowledge exchange framework: Understanding parameters and the capacity for transformative engagement'. Studies in Higher Education, 1-18. https://doi.org/10.1 080/03075079.2020.1735333.

Kirby, D. (2010) 'The future is now: Diegetic prototypes and the role of popular films in generating real-world technological development'. Social Studies of Science, 40 (1), 41-70. https://doi.org/10.1177/0306312709338325.

Lai, I.K. and Lu, T.-W. (2016) 'How to improve the university-industry collaboration in Taiwan's animation industry? Academic vs. industrial perspectives'. Technology Analysis \& Strategic Management, 28 (6), 717-32. https://doi.org/10.1080/09537325.2016.1141404.

Laurel, B. (2013) Computers as Theatre. Upper Saddle River, NJ: Addison-Wesley.

Lee, K.-J. (2011) 'From interpersonal networks to inter-organizational alliances for university-industry collaborations in Japan: The case of the Tokyo Institute of Technology'. R\&D Management, 41 (2), 190-201. https://doi.org/10.1111/j.1467-9310.2011.00633.x.

Levine, D. (2017) 'Design fiction'. DXD, 13 March. Accessed 15 June 2021. https://medium.com/ digital-experience-design/design-fiction-32094e035cd7.

Lindley, J. and Coulton, P. (2016) 'Pushing the limits of design fiction: The case for fictional research papers'. In Proceedings of the 2016 CHI Conference on Human Factors in Computing Systems, 4032-43. https://doi.org/10.1145/2858036.2858446.

Lindley, J. and Potts, R. (2014) 'A machine learning: An example of HCl prototyping with design fiction'. In Proceedings of the 8th Nordic Conference on Human-Computer Interaction: Fun, fast, foundational, 1081-4. https://doi.org/10.1145/2639189.2670281.

Madary, M. and Metzinger, T.K. (2016) 'Real virtuality: A code of ethical conduct. Recommendations for good scientific practice and the consumers of VR-technology'. Frontiers in Robotics and Al, 3. https://doi.org/10.3389/frobt.2016.00003. 
Marquardt, N. (2017) 'Sketching user experiences: Hands-on course of sketching techniques for $\mathrm{HCl}$ research'. In Proceedings of the $2017 \mathrm{CHI}$ Conference Extended Abstracts on Human Factors in Computing Systems. New York: Association for Computing Machinery, 1261-3. https://doi.org/10.1145/3027063.3027107.

MingJi, J. and Ping, Z. (2014) 'Research on the patent innovation performance of university-industry collaboration based on complex network analysis'. Journal of Business-to-Business Marketing, 21 (2), 65-83. https://doi.org/10.1080/1051712X.2014.903454.

Muscio, A. and Vallanti, G. (2014) 'Perceived obstacles to university-industry collaboration: Results from a qualitative survey of Italian academic departments'. Industry and Innovation, 21 (5), 410-29. https://doi.org/10.1080/13662716.2014.969935.

Myers, B.A. (1998) 'A brief history of human-computer interaction technology'. Interactions, 5 (2), 44-54. https://doi.org/10.1145/274430.274436.

Myoken, Y. (2013) 'The role of geographical proximity in university and industry collaboration: Case study of Japanese companies in the UK'. International Journal of Technology Transfer and Commercialisation, 12 (1-3), 43-61. https://doi.org/10.1504/IJTTC.2013.064170.

Newell, A.F., Morgan, M.E., Gregor, P. and Carmichael, A. (2006) 'Theatre as an intermediary between users and $\mathrm{CHI}$ designers'. In $\mathrm{CHI}$ '06 Extended Abstracts on Human Factors in Computing Systems, 111-16. New York: Association for Computing Machinery. https://doi.org/10.1145/1125451.1125479.

Oculus by Facebook (2021) 'Welcome to the Oculus Safety Centre'. Accessed 15 June 2021. www.oculus.com/safety-center.

Research England (2021) 'Knowledge exchange framework'. Accessed 15 June 2021. https://kef. ac.uk/.

Rogers, Y. (2012) 'HCl theory: Classical, modern, and contemporary'. Synthesis Lectures on HumanCentered Informatics, 5 (2), 1-129. https://doi.org/10.2200/S00418ED1V01Y201205HCI014.

Rybnicek, R. and Königsgruber, R. (2019) 'What makes industry-university collaboration succeed? A systematic review of the literature'. Journal of Business Economics, 89 (2), 221-50. https://doi.org/10.1007/s11573-018-0916-6.

Sato, S. and Salvador, T. (1999) 'Methods \& tools: Playacting and focus troupes: Theater techniques for creating quick, intense, immersive, and engaging focus group sessions'. Interactions, 6 (5), 35-41. https://doi.org/10.1145/312683.312715.

Schein, E.H. (2010) Organizational Culture and Leadership. Chichester: John Wiley \& Sons.

Schepers, S., Dreessen, K. and Zaman, B. (2018) 'Exploring user gains in participatory design processes with vulnerable children'. In Proceedings of the 15th Participatory Design Conference: Short papers, situated actions, workshops and tutorial - Volume 2. New York: Association for Computing Machinery, 1-5. https://doi.org/10.1145/3210604.3210617.

Schofield, T. (2013) 'Critical success factors for knowledge transfer collaborations between university and industry'. Journal of Research Administration, 44 (2), 38-56. Accessed 3 June 2021. https://files.eric.ed.gov/fulltext/EJ1156083.pdf.

Scott, S. (2017) 'Transitions and transcendence of the self: Stage fright and the paradox of shy performativity'. Sociology, 51 (4), 715-31. https://doi.org/10.1177/0038038515594093.

Sedgman, K. (2019) 'Challenges of cultural industry knowledge exchange in live performance audience research'. Cultural Trends, 28 (2-3), 103-17. https://doi.org/10.1080/09548963.2019.161 7930.

Sefelin, R., Tscheligi, M. and Giller, V. (2003) 'Paper prototyping - what is it good for? A comparison of paper- and computer-based low-fidelity prototyping'. In CHI '03 Extended Abstracts on Human Factors in Computing Systems, 778-9. https://doi.org/10.1145/765891.765986.

Seland, G. (2009) 'Empowering end users in design of mobile technology using role play as a method: Reflections on the role-play conduction'. In M. Kurosu (ed.), International Conference on Human Centered Design, 912-21. Berlin: Springer. https://doi.org/10.1007/978-3-642-028069_105.

Seland, G. (2010) 'Role-Play Workshops as a User-Centred Design Method for Mobile IT'. PhD thesis, Norwegian University of Science and Technology. http://hdl.handle.net/11250/252258.

Sengers, P. and Csíkszentmihályi, C. (2003) 'HCl and the arts: A conflicted convergence?' In CHI '03 Extended Abstracts on Human Factors in Computing Systems, New York: Association for Computing Machinery, 876-7. https://doi.org/10.1145/765891.766044.

Senior, T.J., Cooper, R., Dovey, J., Follett, G. and Shiach, M. (2016) Connecting to Innovate: A preliminary report on the achievements of the AHRC knowledge exchange hubs for the creative economy. Bristol: University of the West of England. Accessed 28 August 2018. www.react-hub. org.uk/sites/default/files/publications/Connecting-to-Innovate-Final.pdf. 
Shneiderman, B. (2011) 'Technology-mediated social participation: The next 25 years of $\mathrm{HCl}$ challenges'. In J.A. Jacko (ed.), Proceedings of the 14th International Conference on HumanComputer Interaction: Design and development approaches - Volume Part I, 3-14. Berlin: Springer. Accessed 15 June 2021. https://dl.acm.org/doi/10.5555/2022384.2022386.

Spiegel, J.S. (2018) 'The ethics of virtual reality technology: Social hazards and public policy recommendations'. Science and Engineering Ethics, 24 (5), 1537-50. https://doi.org/10.1007/ s11948-017-9979-y.

Staley, K. (2017) 'Changing what researchers "think and do": Is this how involvement impacts on research?' Research for All, 1 (1), 158-67. https://doi.org/10.18546/RFA.01.1.13.

Starbuck, E. (2001) 'Optimizing university research collaborations'. Research-Technology Management, 44 (1), 40-4. https://doi.org/10.1080/08956308.2001.11671406.

Sterling, B. (2013) 'Patently untrue: Fleshy defibrillators and synchronised baseball are changing the future'. Accessed 17 August 2020. www.wired.co.uk/article/patently-untrue.

Sturdee, M. and Lindley, J. (2019) 'Sketching \& drawing as future inquiry in $\mathrm{HCl}^{\prime}$ '. In Proceedings of the Halfway to the Future Symposium 2019. ACM International Conference Proceeding Series, 1-10. https://doi.org/10.1145/3363384.3363402.

Sturdee, M., Coulton, P. and Alexander, J. (2017) 'Using design fiction to inform shape-changing interface design and use'. The Design Journal, 20 (sup1), S4146-S4157. https://doi.org/10.1080/1 4606925.2017.1352913.

Svanaes, D. and Seland, G. (2004) 'Putting the users center stage: Role playing and low-fi prototyping enable end users to design mobile systems'. In Proceedings of the SIGCHI Conference on Human Factors in Computing Systems. New York: Association for Computing Machinery, 479-86. https://doi.org/10.1145/985692.985753.

Tversky, B. (2002) 'What do sketches say about thinking'. In 2002 AAAI Spring Symposium, Sketch Understanding Workshop, Stanford University, AAAI Technical Report SS-02-08, 148-51. Accessed 15 June 2021. www. aaai.org/Papers/Symposia/Spring/2002/SS-02-08/SS02-08-022.pdf.

Williams, A., Dovey, J., Cronin, B., Garside, P., Flintham, M., Smith, M., Barnett, D., Brooks, R., Boddington, A. and Taylor, F. (2017) The Hidden Story: Understanding knowledge exchange partnerships with the creative economy. Accessed 15 June 2021. https://eprints.kingston.ac.uk/ id/eprint/40320/1/THE-HIDDEN_STORY-REPORT-40320-VoR.pdf.

Zhouyuan, Y. (2016) 'The analysis about the factors of silence in college English classroom'. Studies in Literature and Language, 12 (5), 105-10. https://doi.org/10.3968/8402. 